\title{
Remark on the Continuity of the Density of States of Ergodic Finite Difference Operators
}

\author{
François Delyon * and Bernard Souillard * \\ Department of Mathematics, Caltech, Pasadena, CA 91125, USA
}

\begin{abstract}
We give an elementary proof that for a large class of $d$-dimensional finite difference operators including tight-binding models for electron propagation and models for harmonic phonons with random masses or couplings, the integrated density of states is a continuous function of the energy.
\end{abstract}

Let us first consider the self-adjoint finite difference Schrödinger operator acting on $\ell^{2}\left(\mathbb{Z}^{d}\right)$ defined by

$$
(H \psi)(x)=\sum_{\substack{y \in \mathbb{Z}^{d} \\|y-x|=1}} \psi(y)+V(x) \psi(x), \quad V(x) \in \mathbb{R}, \quad x \in \mathbb{Z}^{d}
$$

(if $V$ is unbounded, the set of sequences $\psi$ with finite support is a core for $H$ ). $P(]-\infty, E[)$ will be the associated spectral projection on the energy interval ]$-\infty, E[$, and $P(\{E\})$ the projection on the eigenspace associated with $E$. We, furthermore, consider a probability measure $\mu$ on the potentials $\{V(x)\}_{x \in \mathbb{Z}^{d}}$, namely, a probability measure $\mathbb{R}^{\mathbb{Z}^{d}}$ with the $\sigma$-algebra generated by cylindrical events, and we suppose $\mu$ to be ergodic with respect to the translations of $\mathbb{Z}^{d}$. It is then known [1] that

$$
\left.\left.k_{\Lambda}(E, H)=\frac{1}{|\Lambda|} \operatorname{Tr} P(]-\infty, E\right]\right) \chi_{\Lambda},
$$

where $\chi_{\Lambda}$ stands for the characteristic function of a finite subset $\Lambda$ of $\mathbb{Z}^{d}$, converges as $\Lambda \uparrow \mathbb{Z}^{d}$ for $\mu$-a.a. potential $\{V(x)\}_{x \in \mathbb{Z}^{d}}$ to a non-random function

$$
\left.\left.k(E)=\mathbb{E}_{\mu}\left(\left\langle\delta_{0}, P(]-\infty, E\right]\right) \delta_{0}\right\rangle\right),
$$

where $\mathbb{E}_{\mu}$ denotes expectation with respect to the measure $\mu$ and $\delta_{0}$ is the element located at 0 of the canonical basis of $\ell^{2}\left(\mathbb{Z}^{d}\right) ; k(E)$ is the integrated density of states and can also be obtained by limits of systems enclosed in finite boxes.

* Permanent address: Centre de Physique Théorique, Ecole Polytechnique, F-91128 Palaiseau Cedex, France 
The continuity of $k(E)$ is equivalent to the vanishing of

$$
\mathbb{E}_{\mu}\left(\left\langle\delta_{0}, P(\{E\}) \delta_{0}\right\rangle\right)
$$

which itself, again using ergodicity, is for $\mu$-a.a. potential equal to

$$
\lim _{\Lambda \uparrow \mathbb{Z}^{d}} \frac{1}{|\Lambda|} \operatorname{Tr}\left[P(\{E\}) \chi_{\Lambda}\right] .
$$

When $d=1$, the rank of $P(\{E\}) \chi_{A}$ is less or equal to 2 and the continuity of $k(E)$ follows [1]. The question of the continuity of $k(E)$ for $d>1$ was open until [2], in which log Hölder continuity was proven. We show here that continuity at least can be recovered by a simple proof.

Continuity can, in fact, be recovered by the following argument: note that

$$
\operatorname{dim} \text { Range } \chi_{\Lambda} P(\{E\})<|\partial \Lambda|,
$$

where $\partial \Lambda$ denotes the elements of $\Lambda$ at distance less than 2 from $\mathbb{Z}^{d} \backslash \Lambda$; indeed given an energy $E$ and given $\psi(x)$ for $x \in \partial \Lambda, \psi$ inside $\Lambda$ is uniquely determined by the equation $H \psi=E \psi$. On the other hand,

$$
\begin{aligned}
\frac{1}{|\Lambda|} \operatorname{Tr} P(\{E\}) \chi_{\Lambda} & \leqq \frac{1}{|\Lambda|} \operatorname{dim} \text { Range } P(\{E\}) \chi_{\Lambda} \\
& \leqq \frac{|\partial \Lambda|}{|\Lambda|} . \text { Q.E.D. }
\end{aligned}
$$

Note that the above hypothesis on the measure $\mu$ excludes the cases, where the potential could be infinite at a given site $x$ with a non-zero probability. This is for example the case in the quantum percolation model, for which the potential takes independently at each site the value 0 or $+\infty$ with respective probabilities $p$ and $1-p$; it is easy to check that the integrated density of states is then actually discontinuous.

Our proof extends readily to various other finite difference operators, in particular to the other tight-binding models for electron propagation with diagonal and off-diagonal disorder and to the various models for harmonic phonons with random couplings or random masses. This means operators defined by

$$
(H \psi)(x)=\frac{1}{m(x)} \sum_{\substack{y \in \mathbb{Z}^{d} \\|x-y|=1}} J(x, y) \psi(y), \quad J(x, y)=J(y, x),
$$

acting on the Hilbert space of sequences $\psi(x)$ satisfying $\sum_{x \in \mathbb{Z}^{d}} m(x)|\psi(x)|^{2}<\infty$. The coefficients $\{m(x)\}_{x \in \mathbb{Z}^{d}}$ and $\{J(x, y)\}_{x, y \in \mathbb{Z}^{d},|x-y| \leqq 1}$ are distributed according to a probability measure $\mu$ ergodic with respect to the translations of $\mathbb{Z}^{d}$, and we assume that $\mu$-almost surely the $m(x)$ are strictly positive and the $J(x, y)$ different from zero. This result disagrees with the interpretation sometimes given of numerical computation of the density of states of phonon models [3]. Here again the result holds only under the assumption of ergodicity, but we have excluded the situation, where the coefficients $J(x, y),|x-y|=1$ could be zero with a non-zero probability, in which case the density of states would be discontinuous. 
Simple consequences of the above results are the following:

i) Any given $\lambda$ is almost surely not an eigenvalue of $H$. This follows from our results and Proposition 1 of [1].

ii) If the operators $H$ are periodic, then they have no eigenvalues.

iii) These results extend directly to a large class of hamiltonians with finite range off-diagonal matrix elements.

Acknowledgements. We are glad to thank here W. Luxemburg, B. Simon, and E. Stone for the hospitality of Caltech and B. Simon for twisting our arms until we published this proof.

\section{References}

1. Pastur, L.: Spectral properties of disordered systems in one-body approximation. Commun. Math. Phys. 75, 179 (1980)

2. Craig, W., Simon, B.: Log Hölder continuity of the integrated density of states for stochastic Jacobi matrices. Commun. Math. Phys. 90, 207-218 (1983)

3. We are glad to thank here M. Kac for this remark

Communicated by B. Simon

Received March 1, 1984 
